\title{
BCIO: Brain Computer Interface Ontology
}

\author{
Muhammad Siddiq \\ Department of Computer Science \\ \& IT, Federal Urdu University of \\ Arts, Sciences and Technology, \\ Karachi, Pakistan
}

\author{
Sheikh Kashif Raffat \\ Department of Computer Science \\ \& IT, Federal Urdu University of \\ Arts, Sciences and Technology, \\ Karachi, Pakistan
}

\author{
Farhan Shafiq \\ Department of Computer Science \\ \& IT, Federal Urdu University of \\ Arts, Sciences and Technology, \\ Karachi, Pakistan
}

\begin{abstract}
Brain Computer Interface (BCI) enable paralyze peoples to interact and control their environment by defining the direct communication between human feelings (brain) and technological aspects (external device). The feature extraction and translation of commands in BCI are the critical tasks, which are the key of BCI system. Using the semantic method will enhance the both of these features. Nowadays ontology for representing knowledge is becoming more popular in researchers to describe, share and integrate their scientific data. If the information, algorithms and results are stored in the form of ontological content, it will provide the efficient way to use and reuse the data related to $\mathrm{BCI}$ and will help to $\mathrm{BCI}$ researchers for standardization. Adding semantic in BCI will also improve the efficiency of current BCI system.
\end{abstract}

\section{General Terms}

Human Factor, Brain Computer Interface.

\section{Keywords}

Brain Computer Interface, Ontology, Semantic method.

\section{INTRODUCTION}

Over the few decades, assistive technologies and related research have evaluated to help those people that are unable to operate the environment and perform a task without muscular activation. Brain Computer Interface (BCI) is an example of assistive technology. BCI help "locked-in" state peoples to control their environment by creating communication link between brain and devices without muscular activation [1,2]. The BCI system uses different brain signals recording methods, processing algorithms, protocols and cognitive techniques to extract features from brain signals and translate into the commands those enable devices to perform desired tasks of BCI user.

The semantic model of BCI, that will use the semantic methods to interpreting brain signals, stored in the form of ontological contents will help to produce accurate commands in less time. According to Tom Gruber [3], "Ontology can be viewed as a controlled vocabulary of well-defined terms defined by relationships between them, both human and machine are able to interpretation it easily." The BCI system also required the well-defined vocabulary of symbols and character to translate the signals received from human to perform the desired task. In this paper we proposed an ontology for BCI system that will increase the efficiency of current BCI system.

\section{RELATED WORK}

Magnetic Resonance Images (MRI) is using in neuro-surgery but the functional Magnetic Resonance Images (fMRI) have been used to control the robot in real time trough the brain signals [4]. Mechouche et al. [5] defined that MRI of brain based on ontology in OWL has much significance to assist the surgeon in identification. The feature extraction from image based on ontology was propped by Dameron et al. [6]. The controlled vocabulary of cell signaling was also developed. It's based on signal transduction and attributes of signaling molecules. Wei Wang et al. [7] defined semantic brain computer interface based on Electrocorticographic (ECoG). Carolin Ruf et al. [8] also described that semantic conditions based communication will have more significance in BCI system. The above semantic description of knowledge will help to create the ontology for BCI. The different signals like, Electroiculographic (EOG), Electromyographic (EMG), Electroencephalographic (EEG) etc. are the basic input of BCI system. To handle these signals semantically we studied the "Ontology for Level-One Sensor Fusion" [9], which describe the different signals features. We also studied the Gene Ontology (GO) $[10,11]$ to developed the ontology for BCI system, as GO now became the de facto standard, it will also help to create a standard ontology according to Open Biomedical Ontology (OBO) [12] principles.

\section{BRAIN COMPUTER INTERFACE (BCI)}

Since 1924 when Berger recorded an Electroencephalographic (EEG) to 1973 in which Vidal [13] defined the communication between brain and computer, the field of BCI was opened for researchers. The repaid development was been seen in 1990s $[14,15,16,17]$ when several researchers used different devices and build more comprehensive BCI systems. Invasive, Partially-invasive, Non-invasive BCIs and many toys, based on BCI system are the result of those researches. The basic design of any BCI system can be seen in Figure 1 .

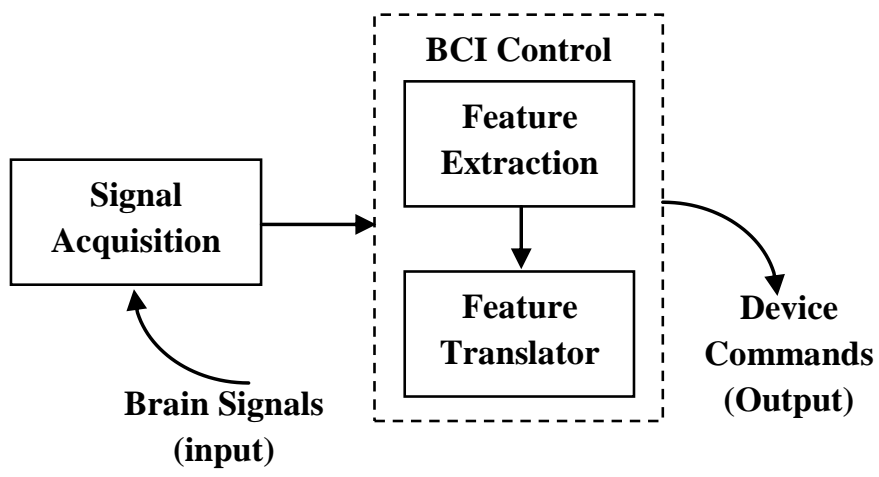

Fig. 1: Basic diagram of BCI system

The BCI2000 [18] is a general purpose BCI system that has the feature like, independence, scalability, real time capabilities, support and facilitation etc. but this existing BCI system model can be modified by adding the semantic approach, using ontology in it. 


\subsection{Ontology for Brain Computer Interface}

The development of ontology for BCI is not a simplest task as it contains the knowledge of different domains. The key feature of ontology development is to analyze domain knowledge. The brain ontology, computer ontology and interface ontology might be the three main ontologies to extract ontology for BCI, as shown in Figure 2.

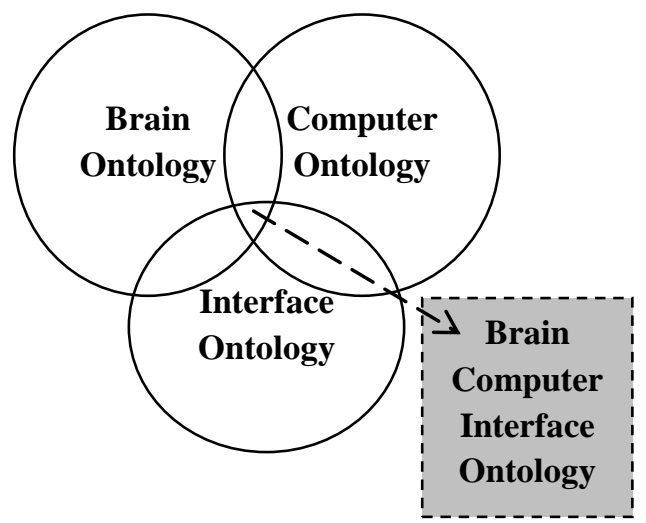

Fig. 2: Block diagram of Brain Computer Interface Ontology

For the development of Brain Computer Interface Ontology (BCIO) the above discussed Gene, Signals, Cell ontologies will also help. We can define a selection operator to extract the feature from these ontologies to develop the ontology for BCI system.

To develop BCIO, we are using OBO-Edit [19] ontology editor and the methodology Ontology Development 101 of Noy [20].

Noy and McGuinness in Ontology Development 101 method, defined seven steps to develop an ontology:

\section{Determine the domain and scope of the ontology}

The domain of BCIO is the different brain signals and the devices used in it. While discussing the scope, we used some relationship to relate $\mathrm{BCIO}$ with other existing ontologies.

\section{Consider reusing existing ontologies}

To reuse existing ontologies, we studied Gene Ontology, Signal Ontology, Cell Signal Ontology, Brain Ontology, Interface Ontology, which can be reuse in BCIO.

\section{Enumerate important terms in the ontology}

We also enumerate signals (terms) according to their properties relevant to the domain of BCIO.

\section{Define the classes and the class hierarchy}

The classes are based on the 'term' defined by different signals and devices.

\section{Define the properties of classes-slots}

To define properties of terms; we will use definition, synonyms (exact, related), comments etc.

\section{Define the facets of the slots}

The facets of slots are the charter, numbers, and symbols that semantically defined in BCIO

\section{Create instances}

The instances are based on the classes 'terms' and the hierarchy of terms, like wave type, pattern, frequency etc.
The all above seven steps are include in development of BCIO iteratively to mature it. The above proposed ontology will use for feature extraction and translation of commands. The Figure 3 is the proposed model of the BCIO. It shows the semantic structure of Brain Computer Interface.

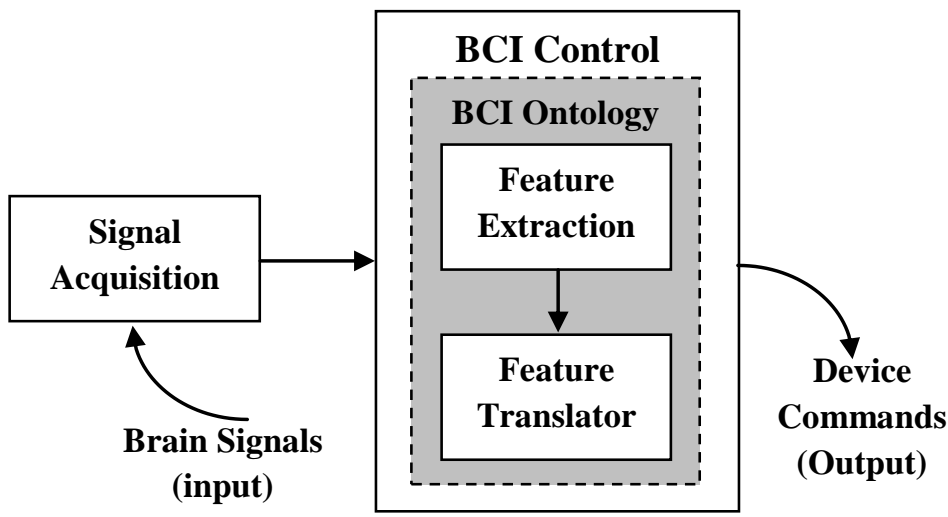

Fig. 3: Basic diagram of BCI system using Ontology

\section{CONCLUSION}

In this paper we proposed the ontological structure of BCI system. Adding semantic will have much significance in any area, specially it will play a vital role in the field of Brain Computer Interface. The key feature of any BCI system is to evaluate and compare the input signals received from brain and process these signals to create commands. These both task will efficiently done by Brain Computer Interface Ontology.

\section{REFERENCES}

[1] A. Kubler, and N. Neumann, "Brain-computer interfacesthe key for the conscious brain locked into a paralyzed body," Progress in Brain Research 150, 513-525, 2005.

[2] M. Kaur, P. Ahmed, and Q. M. Rafiq, "Technology Development for Unblessed People using BCI: A Survey," International Journal of Computer Applications 40(1), 18-24, 2012.

[3] T. Gruber, "Ontology," Entry in the Encyclopedia of Database Systems, Ling Liu and M. Tomer Ozsu (Eds.), Springer-Verlag, 2008.

[4] "To operate robot only with brain," Advanced Telecommunications Research and Honda develop BMI base technology, Tech-on, 26 May 2006.

[5] A, Mechouche, C. Golbreich, and B. Gibaud, "Semantic description of brain MRI images," SWAMM 2006 Workshop, Edinburgh, Irlande, 2006.

[6] O. Dameron, C. Golbreich, O. Bierlaire, and B. Gibaud, "Use case: Ontology with rules for identifying brain anatomical structures," W3C Workshop on Rule Languages for Interoperability, Washington, 2005.

[7] W. Wang, A. D. Degenhart, G.P. Sudre, D.A. Pomerleau, E. C. Tyler-Kabara, "Decoding semantic information from human electrocorticographic (ECoG) signals," Conf Proc IEEE Eng Med Biol Soc. 2011.

[8] C. A. Ruf, A. Furdea, T. Matuz,, D. De Massari, S. Halder, L. Van Der Heiden, B. Kotchoubey, and, N. 
Birbaumer, "Paralysis and Brain-Computer Interface: Classical semantic conditioning for communication?," Conf Comput. Neurosci. \& Neurotech. Bernstein, 2011.

[9] M. G. Ceruti, "Ontology for Level-One Sensor Fusion and Knowledge Discovery," 9th International Conference on Information Fusion 2006, Florence , 2006.

[10] The Gene Ontology Consortium, "The Gene Ontology in 2010," Nucleic Acids Research 38, 2010, doi: 10.1093/nar/gkp1018, D331-D335.

[11] The Gene Ontology Consortium, "The Gene Ontology project in 2008," Nucleic Acids Research 36, 2008, doi: 10.1093/nar/gkm883.

[12] B. Smith, M. Ashburner, C. Rosse, J. Bard, W. Bug, W. Ceusters, L.J. Goldberg, K. Eilbeck, A. Ireland, C.J. Mungall et al. "The OBO Foundry: Coordinated evolution of ontologies to support biomedical data integration," Nature Biotechnology 25, 2007, doi:10.1038/nbt1346.

[13] J. J. Vidal, "Toward direct brain-computer communication," Annual review of Biophysics and Bioengineering 2, 157-180, 1973.

[14] J. R. Wolpaw, D. J. McFarland, G. W. Neat, and C. A. Forneris, "An EEG-based brain-computer interface for cursor control," Electroenceph.Clin. Neurophysiol 78, 252-259, 1991.

[15] E. E. Sutter, "The brain response interface: Communication through visually guided electrical brain responses," J. Microcomput. Applicat 15, 31-45, 1992.

[16] G. Pfurtscheller, D. Flotzinger, and J. Kalcher, "Braincomputer interface-A new communication device for handicapped persons," J. Microcomput.Applicat 16, 293-299, 1993

[17] M. A. Lebedev, M. A. Nicolelis, "Brain-machine interfaces: past, present and future," Trends in Neurosciences 29, 536-546, 2006.

[18] G. Schalk, D. J. McFarland, T. Hinterberger, N Birbaumer, and J. R.Wolpaw, "BCI2000: A GeneralPurpose Brain-Computer Interface (BCI) System," IEEE Transactions on Biomedical Engineering 51, 2004.

[19] J. Day-Richter, M.A. Harris, M. Haendel, The Gene Ontology OBO-Edit Working Group and S. Lewis, "OBO-Edit - an ontology editor for biologists," Bioinformatics 32, Pages 2198-2200, 2007, doi:10.1093.

[20] N.F, Noy and D.L. McGuinness, "Ontology Development 101: A guide to creating your first ontology," Stanford Knowledge Systems Laboratory Technical Report 2001, KSL-01-05, Stanford University, USA. 